\section{Cell differentiatio}

\section{Hepatocytes from non- hepatic adult stem cells}

$(1 \mathrm{t}$ tem cells are undifferentiated longlived cells that are capable of many rounds of division. Here we show that adult human liver cells can be derived from stem cells originating in the bone marrow or circulating outside the liver, raising the possibility that blood-system stem cells could be used clinically to generate hepatocytes for replacing damaged tissue.

Serial transplantation in mice has indicated that some hepatocytes have the stemcell-like property of massive division potential $^{1}$, suggesting that hepatocytes themselves are the principal progenitor cells for new hepatocytes. Also, when hepatocyte regeneration is compromised after injury, facultative stem cells in the bile ducts are activated, producing so-called oval cells that eventually differentiate into hepatocytes ${ }^{2}$.

Cross-gender and whole-liver transplantation studies in rodents indicate that bonemarrow-derived or extrahepatic stem cells can differentiate into hepatocytes ${ }^{3,4}$, so we investigated whether adult human haematopoietic stem cells could contribute to the regeneration of hepatocytes in damaged human liver tissue. First, we tested livers of female patients $(n=9)$ who had received a bone-marrow transplant from a male donor for cells of donor origin by using a DNA probe specific for the centromere of the Y chromosome $e^{5,6}$. Second, we looked for Y-chromosome-positive hepatocytes in female livers grafted into male patients $(n=11)$ that were later removed because of recurrent disease. Y-chromosome-positive hepatocytes should indicate an extrahepatic origin for these cells in both cases.

Our probe detected the Y chromosome in most cells of all types in male control livers (Fig. 1a, b); as expected, inflammatory cells in the transplanted female liver in male recipients were Y-chromosome-positive and so served as a positive control (Fig. 1c). Protease digestion used as part of the Ychromosome detection procedure caused some loss of morphology (Fig. 1d), but hepatocytes were still recognizable by their immunoexpression of cytokeratin 8 (ref. 7): many were Y-chromosome-positive, indicating that they originated from maledonor bone marrow.

It has been suggested that microchimaerism for Y-chromosome-positive cells occurs in the human female liver as a result of the transplacental passage of male fetal blood cells during pregnancy ${ }^{8}$. However, we did not detect any Y-chromosomepositive leukocytes or hepatocytes ${ }^{9}$ in liver biopsies taken from such women in a fluorescence in situ hybridization (FISH) assay. Although differentiation of fetal

Figure 1 Photomicrographs showing the presence of the $Y$ chromosome, detected by immunolocalization of a fluorescein isothiocyanate (FITC)-labelled Y-chromosome probe using an anti-fluorescein antibody conjugated to horseradish peroxidase, and visualized using diaminobenzidine as a brown chromogen. The presence of the $Y$ chromosome is indicated by a distinct brown dot, typically located at the nuclear periphery. a, Hepatocytes were identified by their large round nuclei and cytoplasmic granules and were $Y$ chromosome-positive in male controls. b, The $Y$ chromosome was also detected in the bile ducts (D) in male controls. c, A Ychromosome-negative bile

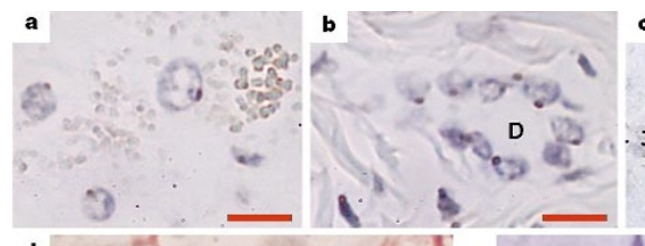

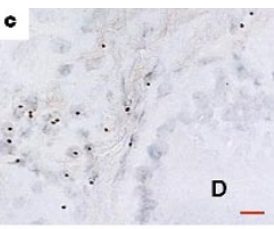

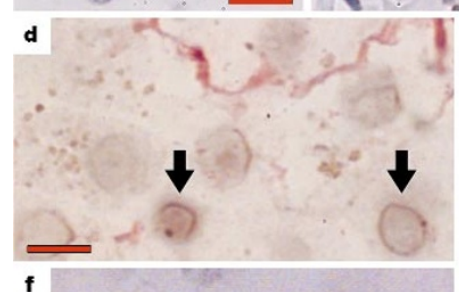
e ह
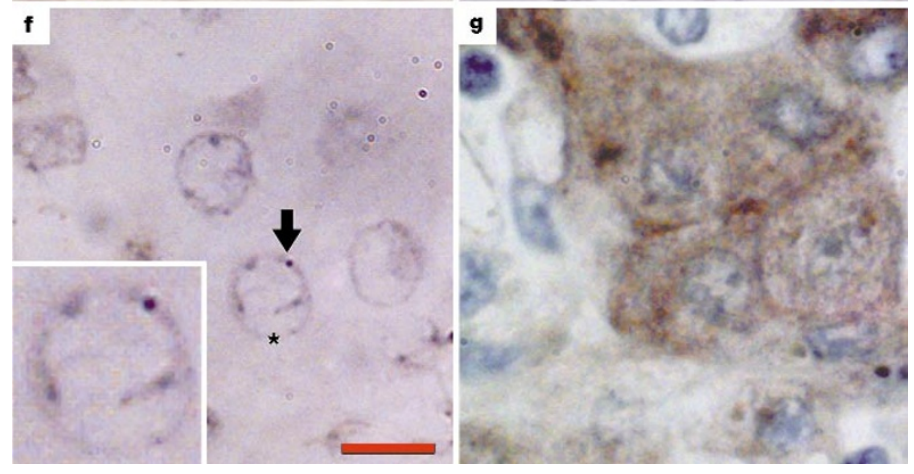

duct surrounded by numerous Y-positive inflammatory cells in a female liver transplanted into a male. d, Immunodetection of two Y-chromosome-positive cells (arrows) located in a hepatocyte plate delineated by cytokeratin-8 immunostaining (red) from a female patient who had received male bone marrow. $\mathbf{e}-\mathbf{g}, \mathrm{Y}$-chromosome detection in female livers transplanted into male recipients; $\mathbf{e}$, two Y-chromosome-positive hepatocytes (arrows) in a hepatocyte plate bordering a sinusoid (S); note the brown dot, demonstrating that the $Y$ chromosome is readily distinguishable from the blue nucleolus; $\mathbf{f}, \mathbf{g}$, consecutive sections of a group of four hepatocyte nuclei, showing $\mathbf{f}$, one $\mathbf{Y}$-chromosome-positive hepatocyte (arrow, and inset at 2-fold magnification), and $\mathbf{g}$, their cytokeratin-8 immunoreactivity. Scale bars, $10 \mu \mathrm{m}$.

bone-marrow stem cells into hepatocytes might occur after placental transfer, this is unlikely in the patient whose liver biopsy is shown in Fig. 1d because there was no history of male childbearing or abortion.

Chronic damage in the livers of our bone-marrow-transplant patients could promote the colonization and amplification of exogenous haematopoietic stem cells, providing a rationale for experimental models of hepatocyte transplantation in which blocking regeneration by indigenous cells or promoting their apoptosis encourages growth of transplanted cells in the damaged liver ${ }^{10,11}$.

So do an individual's haematopoietic stem cells mobilize during liver failure to increase the regenerative capacity of their liver? We found examples of Y-chromosomepositive epithelial cells whose identity as hepatocytes was confirmed by their location and expression of cytokeratin 8 (Fig. 1e-g), indicating that circulating extrahepatic stem cells of endogenous, as well as exogenous, origin, can colonize the liver. Our liver samples were each from unique clinical cases, requiring individual protease digestion; the frequency of Y-chromosome-positive hepatocytes was relatively low in all of them (about 0.5-2\%) and they often appeared as clusters (Fig. 1d, e), as though clonal growth had occurred after colonization.

Haematopoietic stem cells can be readily harvested from an individual, and we have shown that the human adult haematopoiet- ic stem-cell population is capable of being instructed by the appropriate environment to yield an epithelial lineage. Our results should contribute to the development of human tissue for use in a therapeutic context.

Malcolm R. Alison ${ }^{\star}$, Richard Poulsom $†$,

Rosemary Jeffery $\dagger$, Amar P. Dhillon $\ddagger$,

Alberto Quaglia $\ddagger$, Joe Jacob*,

Marco Novelli§, Grant Prentice\|, Jill Williamsong, Nicholas A. Wright ${ }^{\star} \dagger$

${ }^{\star}$ Department of Histopathology, Imperial College

School of Medicine, Hammersmith Campus,

London W12 0NN, UK

e-mail:m.alison@ic.ac.uk

$\dagger$ Histopathology Unit, Imperial Cancer Research

Fund, London WC2A 3PX, UK

$\ddagger$ Departments of Histopathology and

IHaematology, Royal Free Campus, Royal Free and

University College School of Medicine, London

NW3 $2 P F, U K$

$\$$ Department of Histopathology, University College London, London WC1E 6JJ, UK

Guman Cytogenetics Laboratory, Imperial Cancer Research Fund, London WC2A 3PX, UK

1. Overturf, K. et al. Am. J. Pathol. 151, 1273-1280 (1997).

2. Alison, M. R. Curr. Opin. Cell Biol. 10, 710-715 (1998)

3. Petersen, B. E. et al. Science 284, 1168-1170 (1999).

4. Theise, N. D. et al. Hepatology 31, 235-240 (2000).

5. Wolfe, J. et al. J. Mol. Biol. 182, 477-485 (1985).

6. Novelli, M. R. et al. Science 272, 1187-1190 (1996).

7. Van Eyken, P. \& Desmet, V. J. Liver 13, 113-122 (1993).

8. Tanaka, A. et al. Hepatology 30, 833-838 (1999).

9. Rubbia-Brandt, L. et al. Hepatology 30, 821-822 (1999).

10. Laconi, E. et al. Am. J. Pathol. 153, 319-329 (1998).

11. Oren, R. et al. Hepatology 30, 903-913 (1999). 